\title{
The swampland conjecture and the Higgs expectation value
}

\author{
Koichi Hamaguchi, ${ }^{a, b}$ Masahiro Ibe ${ }^{b, c}$ and Takeo Moroi ${ }^{a, b}$ \\ ${ }^{a}$ Department of Physics, Faculty of Science, The University of Tokyo, \\ Bunkyo-ku, Tokyo 113-0033, Japan \\ ${ }^{b}$ Kavli IPMU (WPI), UTIAS, The University of Tokyo, \\ Kashiwa, Chiba 277-8583, Japan \\ ${ }^{c}$ ICRR, The University of Tokyo, \\ Kashiwa, Chiba 277-8582, Japan \\ E-mail: hama@hep-th.phys.s.u-tokyo.ac.jp, ibe@icrr.u-tokyo.ac.jp, \\ moroi@phys.s.u-tokyo.ac.jp
}

ABSTRACT: The recently proposed de Sitter swampland conjecture excludes local extrema of a scalar potential with a positive energy density in a low energy effective theory. Under the conjecture, the observed dark energy cannot be explained by the cosmological constant. The local maximum of the Higgs potential at the symmetric point also contradicts the conjecture. In order to make the Standard Model consistent with the conjecture, it has been proposed to introduce a quintessence field, $Q$, which couples to the cosmological constant and the local maximum of the Higgs potential. In this paper, we show that such a modified Higgs potential generically results in a $Q$-dependent Higgs vacuum expectation value (VEV). The $Q$-dependence of the Higgs VEV induces a long-range force, which is severely excluded by the tests of the equivalence principle. Besides, as the quintessence field is in motion, the Higgs VEV shows a time-dependence, which is also severely constrained by the measurements of the time-dependence of the proton-to-electron mass ratio. Those constraints require an additional fine-tuning which is justified neither by the swampland conjecture nor the anthropic principle. We further show that, even if such an unjustified fine-tuning condition is imposed at the tree level, radiative corrections upset it. Consequently, we argue that most of the habitable vacua in the string landscape are in tension with the phenomenological constraints.

Keywords: Effective Field Theories, Renormalization Group, Renormalization Regularization and Renormalons

ArXiv EPrint: 1810.02095 


\section{Contents}

1 Introduction 1

2 Salvaging the Higgs potential from swampland 2

2.1 Higgs potential with a tiny dark energy 2

2.2 Constraints on the quintessence dependent Higgs VEV 5

$\begin{array}{lll}2.2 .1 & \text { Long-range force } & 6\end{array}$

2.2.2 Time-varying electron-to-proton mass ratio 6

$\begin{array}{lll}2.2 .3 & \text { Unjustified fine-tuning } & 7\end{array}$

3 Radiatively induced $Q$-dependent Higgs VEV $\quad 8$

3.1 Wilsonian approach 8

$\begin{array}{ll}3.2 & \text { Analysis in the } \overline{\mathrm{MS}} \text { prescription } \\ 3.3\end{array}$

$\begin{array}{lll}3.3 & \text { Another constraint } & 15\end{array}$

4 Conclusions and discussions $\quad 16$

$\begin{array}{lr}\text { A Time evolution of quintessence field } & 18\end{array}$

\section{Introduction}

As the string theory is virtually a unique candidate for a theory of quantum gravity, the consistency between a low energy effective field theory and the string theory is a prime concern for physics beyond the Standard Model. So far, several consistency conditions have been conjectured from the string theory [1-4]. Low-energy effective field theories which do not satisfy those conjectures are said to be not in the string landscape, but in the swampland and are disfavored.

Among various conjectures, the most recent one [4], the so-called de Sitter swampland conjecture, stimulates intensive studies of its phenomenological and cosmological consequences [5-36]. Under the conjecture, the scalar potential of a set of scalar fields $\{\phi\}$, $V_{\text {total }}$, satisfies the condition,

$$
M_{\mathrm{PL}}\left|\nabla V_{\text {total }}\right|>c V_{\text {total }} \text {. }
$$

Here, $c$ is a positive constant of $\mathcal{O}(1)$ and $M_{\mathrm{PL}}$ is the reduced Planck scale. The size of the potential gradient, $\left|\nabla V_{\text {total }}\right|$, is given by

$$
\left|\nabla V_{\text {total }}\right|=\left(\sum_{\phi}\left(\partial V_{\text {total }} / \partial \phi\right)^{2}\right)^{1 / 2}
$$

for the canonically normalized scalar fields.

Immediate consequences of the conjecture are 
- The observed dark energy cannot be explained by a positive cosmological constant.

- The local maximum of the Higgs potential at the symmetric point, $H=0$, is inconsistent with the conjecture.

As discussed in refs. $[4,13,26]$, the most straightforward resolution of these tensions is to couple the cosmological constant and the Higgs potential to the so-called quintessence field $Q$, whose coupling is suppressed by the Planck scale [37-39].

In this paper, we show that such a modified Higgs potential generically predicts a $Q$-dependent vacuum expectation value $(\mathrm{VEV})$ of the Higgs field. The $Q$-dependence of the Higgs VEV induces a long-range force which is severely excluded by the tests of the equivalence principle [40]. In addition, the $Q$-dependence results in a time-dependent Higgs VEV. We show that the precise spectroscopic measurements of the proton-to-electron mass ratio in distant astrophysical systems [41, and references therein] put stringent constraint on the time-varying Higgs VEV. Consequently, we argue that most of the habitable vacua in the string landscape are in tension with the phenomenological constraints unless there is an additional fine-tuning that is justified neither by the swampland conjecture nor the anthropic principle. We further show that, even if such an unjustified fine-tuning condition is imposed at the tree level, it is inevitably violated by radiative corrections. Therefore, under the de Sitter swampland conjecture, most of the habitable vacua in the string landscape contradict with the observations.

The organization of the paper is as follows. In section 2, we first discuss how we can retrofit the Higgs potential so that it can be salvaged from the swampland at the tree level argument. We then show that, such a modified potential generically leads to a $Q$ dependent Higgs VEV, which is severely constrained by phenomenological requirements. We argue that an additional fine-tuning condition is required, which is justified neither by the swampland conjecture nor the anthropic principle. In section 3 , we study radiative corrections to the $Q$-dependent Higgs potential, and show that they generically upset the fine-tuning condition imposed at the tree level. The final section is devoted to our conclusions. In appendix A, we give a rough estimate of the excursion of the quintessence field from the early universe to the present.

\section{Salvaging the Higgs potential from swampland}

\subsection{Higgs potential with a tiny dark energy}

The most straightforward way to make the observed dark energy consistent with the de Sitter swampland conjecture is to introduce the so-called quintessence field [4]. Here, we take the simplest form of the potential of the (real-valued) quintessence field, $Q$, in the present universe (i.e., in the universe after the electroweak phase transition):

$$
V_{Q}(Q)=3 \xi_{c c} H_{0}^{2} M_{\mathrm{PL}}^{2} e^{-c_{Q} Q / M_{\mathrm{PL}}},
$$

where $H_{0}$ is the expansion rate of the present universe, while $\xi_{c c}$ and $c_{Q}$ are positive-valued constant parameters. ${ }^{1}$ We set $Q=0$ as the present value without loss of generality. Then,

\footnotetext{
${ }^{1}$ For $c_{Q}<0$, we redefine $Q^{\prime}=-Q$
} 
$\xi_{c c}$ is set to be $\xi_{c c} \simeq \Omega_{\mathrm{DE}} \simeq 0.7$ [42] (with $\Omega_{\mathrm{DE}}$ being the density parameter of the dark energy) to explain the observed dark energy density (see appendix A for more details). The potential does not have any local extrema with a positive energy density, and hence it satisfies the swampland conjecture for $c_{Q}=\mathcal{O}(1) .{ }^{2}$ It should be noted that the following arguments do not depend on the details of $V_{Q}(Q)$ as long as it satisfies the swampland conjecture.

Now, let us discuss how the swampland conjecture restricts the Higgs sector. The potential for the Higgs field in the Standard Model is given by

$$
V_{H}(H)=-M_{H}^{2}|H|^{2}+\lambda|H|^{4}+\Lambda_{\mathrm{EW}}^{4},
$$

where $M_{H}^{2}>0$ is a squared Higgs mass parameter and $\lambda>0$ the Higgs quartic coupling constant. A cosmological constant parameter, $\Lambda_{\mathrm{EW}}^{4}$, is required so that the vacuum energy is cancelled at the Higgs vacuum expectation value, ${ }^{3}$

$$
\langle H\rangle^{2}=\frac{v_{H}^{2}}{2}=\frac{M_{H}^{2}}{2 \lambda},
$$

where the fine-tuning condition is

$$
V_{H}(\langle H\rangle)=-\frac{M_{H}^{4}}{4 \lambda}+\Lambda_{\mathrm{EW}}^{4} \simeq 0 .
$$

As pointed out in $[13,26]$, the Higgs potential in eq. (2.2) does not satisfy the de Sitter swampland conjecture at the symmetric point, $H=0$. In fact, the left-hand side of eq. (1.1),

$$
M_{\mathrm{PL}}\left|\nabla V_{\text {total }}\right|_{H=0}=M_{\mathrm{PL}}\left|\partial V_{Q} / \partial Q\right|=3 c_{Q} \xi_{c c} H_{0}^{2} M_{\mathrm{PL}}^{2} e^{-c_{Q} Q / M_{\mathrm{PL}}},
$$

is much smaller than the right-hand side,

$$
\left.V_{\text {total }}\right|_{H=0} \simeq V_{H}(H=0)=\Lambda_{\mathrm{EW}}^{4} \simeq \frac{M_{H}^{4}}{4 \lambda} \gg H_{0}^{2} M_{\mathrm{PL}}^{2} .
$$

An immediate remedy to make the Higgs potential consistent with the de Sitter swampland conjecture is to retrofit the $\Lambda_{\mathrm{EW}}^{4}$ term to couple to the quintessence field, i.e.,

$$
V_{H}^{(a)}(H, Q)=-M_{H}^{2}|H|^{2}+\lambda|H|^{4}+\Lambda_{\mathrm{EW}}^{4} e^{-c_{H} Q / M_{\mathrm{PL}}},
$$

with $c_{H}=\mathcal{O}(1)$. With the modification, the left-hand side of eq. (1.1) becomes

$$
M_{\mathrm{PL}}\left|\nabla V_{\text {total }}\right|_{H=0} \simeq\left|\partial V_{H}^{(a)} / \partial Q\right|_{H=0}=\left|c_{H}\right| \Lambda_{\mathrm{EW}}^{4} e^{-c_{H} Q / M_{\mathrm{PL}}},
$$

which is comparable with the right-hand side,

$$
\left.V_{\text {total }}\right|_{H=0} \simeq \Lambda_{\mathrm{EW}}^{4} e^{-c_{H} Q / M_{\mathrm{PL}}} .
$$

\footnotetext{
${ }^{2}$ We assume that the quintessence field satisfies the slow-role condition, $c_{Q}<\sqrt{6}$.

${ }^{3}$ Here, $\langle H\rangle$ denotes the VEV of the second component of the Higgs doublet.
} 
In this way, the Higgs potential in eq. (2.7) can be consistent with the de Sitter swampland conjecture.

The modified Higgs potential in eq. (2.7), however, has a serious problem. At the present vacuum, the fine-tuning condition of the vacuum energy is given by

$$
V_{H}^{(a)}(H=\langle H\rangle, Q=0)=-\frac{M_{H}^{4}}{4 \lambda}+\left.\Lambda_{\mathrm{EW}}^{4} e^{-c_{H} Q / M_{\mathrm{PL}}}\right|_{Q=0} \simeq 0
$$

However, the quintessence field feels a strong potential force from the coupling to $\Lambda_{\mathrm{EW}}^{4}$ at the present vacuum,

$$
\left.\left|\partial V_{H}^{(a)} / \partial Q\right|\right|_{H=\langle H\rangle}=\left|c_{H}\right| \frac{\Lambda_{\mathrm{EW}}^{4}}{M_{\mathrm{PL}}} e^{-c_{H} Q / M_{\mathrm{PL}}},
$$

which makes the quintessence field moves from $Q=0$. Accordingly, the fine-tuning condition in eq. (2.10) is immediately violated once the quintessence field evolves in time, within a time scale of $\tau \sim M_{\mathrm{PL}} / \Lambda_{\mathrm{EW}}^{2} \sim O\left(10^{-10}\right)$ sec. $^{4}$ Therefore, although the Higgs potential in eq. (2.7) is consistent with the swampland conjecture, it is not habitable, and hence, does not satisfy the anthropic principle [43-45].

In order to avoid this problem, the Higgs potential needs to be further modified so that the fine-tuning condition of the vacuum energy at $H=\langle H\rangle$ is not affected by the motion of the quintessence field. Such a requirement can be satisfied, for example, by extending the quintessence-Higgs coupling to

$$
V_{H}^{(b)}(H, Q)=-M_{H}^{2} e^{-c_{M} Q / M_{\mathrm{PL}}}|H|^{2}+\lambda e^{-c_{\lambda} Q / M_{\mathrm{PL}}}|H|^{4}+\Lambda_{\mathrm{EW}}^{4} e^{-c_{H} Q / M_{\mathrm{PL}}},
$$

where $c_{M}$ and $c_{\lambda}$ are $\mathcal{O}(1)$ coefficients. For a given value of the quintessence field $Q$, the Higgs VEV is then given by

$$
\left.\langle H\rangle^{2}\right|_{V_{H}^{(b)}}=\frac{v_{H}(X)^{2}}{2}=\frac{M_{H}^{2} e^{-\left(c_{M}-c_{\lambda}\right) X}}{2 \lambda},
$$

where $X=Q / M_{\mathrm{PL}}$. As a result, the fine-tuning condition of the vacuum energy is given by

$$
V_{H}^{(b)}(\langle H\rangle, Q)=\left(-\frac{M_{H}^{4}}{4 \lambda} e^{-\left(2 c_{M}-c_{\lambda}-c_{H}\right) Q / M_{\mathrm{PL}}}+\Lambda_{\mathrm{EW}}^{4}\right) e^{-c_{H} Q / M_{\mathrm{PL}}} \simeq 0 .
$$

Therefore, the stability of the small dark energy is achieved by imposing a fine-tuning condition,

$$
2 c_{M}-c_{\lambda}-c_{H}=0
$$

In this way, we arrive at a Higgs potential which is consistent with the de Sitter swampland conjecture and the anthropic principle. It should be stressed that the additional fine-tuning condition in eq. (2.15) for the stability of the small vacuum energy does not make the model less plausible, since we anyway need to find habitable vacua in the string landscape [43-45].

\footnotetext{
${ }^{4}$ In this case, the quintessence field evolves so rapidly that the observed current Universe is never realized.
} 


\subsection{Constraints on the quintessence dependent Higgs VEV}

A crucial feature of the Higgs potential, $V_{H}^{(b)}$, is that the Higgs VEV generically depends on the quintessence field, as shown in (2.13). This $Q$-dependent Higgs VEV induces effective Yukawa couplings between the quintessence field and the matter fields in the Standard Model, ${ }^{5}$

$$
\left.\mathcal{L}_{\text {eff }} \simeq \sum_{\substack{i=\text { quarks } \\ \text { leptons }}} \frac{m_{i}}{M_{\mathrm{PL}}} \frac{d \ln v_{H}(X)}{d X}\right|_{X=0} Q \bar{\psi}_{i} \psi_{i}
$$

with

$$
\left.\frac{d \ln v_{H}(X)}{d X}\right|_{X=0}=-\frac{1}{2}\left(c_{M}-c_{\lambda}\right) .
$$

Here, $m_{i}$ denotes the mass of the corresponding fermion.

The coupling of the quintessence field to the quarks leads to its coupling to the nucleons [46],

$$
\mathcal{L}_{\text {eff }}=\left.\frac{m_{N} f_{N}}{M_{\mathrm{PL}}} \frac{d \ln v_{H}(X)}{d X}\right|_{X=0} Q \overline{\psi_{N}} \psi_{N},
$$

where $m_{N}$ is the nucleon mass, and $f_{N}$ is defined as

$$
f_{N}=\sum_{q=u, d, s, c, b, t} f_{q}^{N}=\frac{2}{9}+\frac{7}{9} \sum_{q=u, d, s} f_{q}^{N},
$$

with

$$
f_{q}^{N}=\frac{1}{m_{N}}\left\langle N\left|m_{q} \bar{\psi}_{q} \psi_{q}\right| N\right\rangle .
$$

In the following, we use the scalar coupling estimated by using phenomenological and lattice QCD calculations [47],

$$
f_{N}=0.308(18)
$$

The isospin violating effect is also estimated to be,

$$
f_{p}-f_{n} \simeq-1.5 \times 10^{-3},
$$

with an $\mathcal{O}(10) \%$ accuracy [48]. Altogether, we find that the electron and nucleons couple to the quintessence field,

$$
\mathcal{L}_{\mathrm{eff}}=q_{e} Q \bar{\psi}_{e} \psi_{e}+q_{p} Q \bar{\psi}_{p} \psi_{p}+q_{n} Q \bar{\psi}_{n} \psi_{n},
$$

where

$$
\begin{aligned}
q_{e} & =\left.\frac{m_{e}}{M_{\mathrm{PL}}} \frac{d \ln v_{H}(X)}{d X}\right|_{X=0}, \\
q_{p, n} & =\left.\frac{m_{p, n} f_{p, n}^{N}}{M_{\mathrm{PL}}} \frac{d \ln v_{H}(X)}{d X}\right|_{X=0} .
\end{aligned}
$$

\footnotetext{
${ }^{5}$ The effective Yukawa couplings can also be obtained by diagonalizing the mass matrix of the Higgs and quintessence fields, which leads to a mixing between them in the basis of mass eigenstates.
} 


\subsubsection{Long-range force}

The Yukawa interaction of the quintessence field to the electron and the nucleons, eq. (2.23), induces a long range force among electrons and nucleons, which are severely constrained by the tests of the equivalence principle [40]. In our setup, the parameters $\tilde{g}$ and $\tilde{\psi}$ in [40] are identified as

$$
\begin{aligned}
\tilde{g}^{2} & =\left(q_{e}+q_{p}\right)^{2}+q_{n}^{2}, \\
\tan \tilde{\psi} & =\frac{q_{n}}{q_{e}+q_{p}} .
\end{aligned}
$$

The angle $\tilde{\psi}$ is given by $\tilde{\psi} \simeq \pi / 4$ for $q_{e} \ll q_{n} \simeq q_{p}$, and the constraint reads

$$
\sqrt{\left(q_{e}+q_{p}\right)^{2}+q_{n}^{2}} \lesssim 4 \times 10^{-24}
$$

Thus, in the model with the Higgs potential $V_{H}^{(b)}$, we find a stringent constraint,

$$
\left|c_{M}-c_{\lambda}\right| \lesssim 0.4 \times 10^{-4} \text {. }
$$

Consequently, the Higgs potential $V_{H}^{(b)}$ is in tension with observations, although it is consistent with the de Sitter swampland conjecture and the anthropic principle.

\subsubsection{Time-varying electron-to-proton mass ratio}

Let us also note that the quintessence field is in motion at present due to the potential force in eq. (2.1), which leads to a non-trivial shift of $Q$. We have numerically solved the cosmological evolution of $Q$, and the results are summarized in appendix A. For example, the shift of $Q$ from $z=1$ to the present (with $z$ being the redshift parameter) is estimated as

$$
\left.\frac{\Delta Q}{M_{\mathrm{PL}}}\right|_{z=1} \simeq-0.24 \times c_{Q}
$$

Accordingly, the masses of the electron and the proton also depend on time through the quintessence couplings in eq. (2.23);

$$
\begin{aligned}
& \left.\frac{\Delta m_{e}}{m_{e}}\right|_{z=1}=\left.\frac{d \ln v_{H}(X)}{d X}\right|_{X=0} \times\left.\frac{\Delta Q}{M_{\mathrm{PL}}}\right|_{z=1}, \\
& \left.\frac{\Delta m_{p}}{m_{p}}\right|_{z=1}=f_{p} \times\left.\frac{d \ln v_{H}(X)}{d X}\right|_{X=0} \times\left.\frac{\Delta Q}{M_{\mathrm{PL}}}\right|_{z=1} .
\end{aligned}
$$

Thus, we find that the ratio of the proton-to-electron mass, $\mu_{p e}=m_{p} / m_{e}$, exhibits a time dependence,

$$
\left.\frac{\Delta \mu_{p e}}{\mu_{p e}}\right|_{z=1} \simeq-0.7 \times\left.\frac{d \ln v_{H}(X)}{d X}\right|_{X=0} \times\left.\frac{\Delta Q}{M_{\mathrm{PL}}}\right|_{z=1} .
$$

Such a time dependence of $\mu_{p e}$ is severely constrained by spectroscopic measurements of distant astrophysical systems. A compilation of the spectroscopic tests [41, and references therein] amounts to

$$
\left.\frac{\Delta \mu_{p e}}{\mu_{p e}}\right|_{z<1}=(-0.24 \pm 0.09) \times 10^{-6} .
$$


Therefore, we find that the constraint on a time-varying proton-to-electron mass ratio leads to

$$
c_{Q}\left|c_{M}-c_{\lambda}\right| \lesssim 0.4 \times 10^{-5}
$$

similarly to the condition obtained from the long-range force constraint (2.29).

So far, we assumed that the field which tilts the local maximum of the Higgs potential (which we denote as $Q_{H}$ here) and the quintessence field of the vacuum energy, $Q$, are the same field. One may argue that the tensions with the tests of the time-varying proton-toelectron ratio can be resolved by assuming that these fields are independent with each other and the motion of the quintessence field does not affect the Higgs VEV at all even if the Higgs VEV depends on $Q_{H}$. However, as we will discuss, not only the tree level contribution but also radiative contributions to the vacuum energy from the Higgs sector are required to be cancelled to explain the tiny observed dark energy. Thus, the coupling of $Q_{H}$ to the Higgs field inevitably results in a coupling of $Q_{H}$ to the dark energy. Therefore, it is generically expected that $Q_{H}$ is also in motion as in the case of $Q$, and hence, the assumption of the independent $Q_{H}$ does not resolve the tension, unless there is a fundamental reason to forbid a time-varying Higgs expectation value.

\subsubsection{Unjustified fine-tuning}

From the phenomenological constraints (2.29) and (2.35), we arrive at an additional finetuning condition

$$
c_{M}-c_{\lambda} \simeq 0
$$

By combining eqs. (2.14), (2.15) and (2.36), the Higgs potential is then restricted to a form

$$
V_{H}^{(c)}(H, Q)=\left(-M_{H}^{2}|H|^{2}+\lambda|H|^{4}+\Lambda_{\mathrm{EW}}^{4}\right) e^{-c_{H} Q / M_{\mathrm{PL}}}=\lambda e^{-c_{H} Q / M_{\mathrm{PL}}}\left(|H|^{2}-\frac{1}{2} v_{H}^{2}\right)^{2}
$$

which corresponds to the Higgs-quintessence coupling proposed in [13]. ${ }^{6}$ As the quintessence coupling is factored out as an overall factor, the Higgs VEV does not depend on the quintessence field.

It should be stressed that the additional fine-tuning in eq. (2.36), or more generically an independence of the Higgs VEV on the quintessence field, is justified neither by the swampland conjecture nor the anthropic principle, but it is required from purely phenomenological reasons. ${ }^{7}$ In other words, under the assumption that the swampland conjecture is satisfied by a quintessence-like field, most of the habitable vacua in the string landscape are excluded by the observational constraints, unless there is an additional fine-tuning that is justified neither by the conjecture nor the anthropic argument.

\footnotetext{
${ }^{6}$ See ref. [49] for related discussions on the Higgs-quintessence couplings.

${ }^{7}$ As discussed in [50], the Higgs VEV in the era of the Big-Bang Nucleosynthesis (BBN) is allowed to be different from the current value by a factor $\mathcal{O}(1)$ for habitable universe. See also [51] for a related discussion.
} 
In principle, it is possible to assume $Q$-dependent Yukawa couplings which make the fermion masses independent of the quintessence field. Again, however, such fine-tunings are not required by the swampland conjecture nor the anthropic principle. Therefore, this possibility does not explain why there is no long-range force nor why the proton-to-electron mass ratio is time-independent. Besides, the $Q$-dependent Yukawa couplings lead to $Q$ dependences of the gauge couplings through radiative corrections, which are also restricted by the tests on the time-varying coupling constants [41, and references therein]. In this paper, we do not pursue these possibilities any further.

\section{Radiatively induced $Q$-dependent Higgs VEV}

As we have discussed in the previous section, it is required to choose Higgs-quintessence couplings so that the Higgs VEV does not depend on the quintessence field, although such a condition is not required from the de Sitter swampland conjecture nor the anthropic principle. The Higgs potential $V_{H}^{(c)}$ in eq. (2.37) is the simplest example which satisfies this condition. In this section, we assume $V_{H}^{(c)}$ and discuss whether the Higgs VEV remains independent of the quintessence field when we consider radiative corrections in the low energy effective field theory.

\subsection{Wilsonian approach}

To obtain a rough idea how the radiative corrections affect the low energy effective field theory, let us first assume that the Higgs potential in a Wilsonian effective action at around the Planck scale is given by $V_{H}^{(c)}$. We also assume that the other couplings such as the gauge coupling constants and the Yukawa coupling constants do not depend on the quintessence field. This assumption is motivated by the fact that the $Q$-dependences of them are severely constrained by the tests of the equivalence principle, by the test of the time-variation of the fundamental couplings, and by the BBN constraints [40, 41, 52].

In this setup, the squared Higgs mass parameter and the quartic coupling and at a low energy scale receives radiative corrections

$$
M_{H}^{2}\left(\mu_{R}\right)=\left.M_{H}^{2} e^{-c_{H} Q / M_{\mathrm{PL}}}\left(1+\int_{M_{\mathrm{PL}}}^{\mu_{R}} \frac{d \mu_{R}^{\prime}}{\mu_{R}^{\prime}} \gamma_{M_{H}^{2}}\left(\mu_{R}^{\prime}\right)\right) \simeq M_{H}^{2}\left(\mu_{R}\right)\right|_{Q=0} e^{-c_{H} Q / M_{\mathrm{PL}}},
$$

and

$$
\begin{aligned}
\lambda\left(\mu_{R}\right) & =\lambda e^{-c_{H} Q / M_{\mathrm{PL}}}+\int_{M_{\mathrm{PL}}}^{\mu_{R}} \frac{d \mu_{R}^{\prime}}{\mu_{R}^{\prime}} \beta_{\lambda}\left(\mu_{R}^{\prime}\right) \\
& \simeq\left[\left.\lambda\left(\mu_{R}\right)\right|_{Q=0}+\left(e^{c_{H} Q / M_{\mathrm{PL}}}-1\right) \int_{M_{\mathrm{PL}}}^{\mu_{R}} \frac{d \mu_{R}^{\prime}}{\mu_{R}^{\prime}} \beta_{\lambda}\left(\mu_{R}^{\prime}\right)\right] e^{-c_{H} Q / M_{\mathrm{PL}}},
\end{aligned}
$$

where $\mu_{R}^{(\prime)}$ denotes the renormalization scale, $\beta_{\lambda}$ the beta functions of $\lambda$, and $\gamma_{M_{H}^{2}}$ the anomalous dimension of $M_{H}^{2}$. In eqs. (3.1) and (3.2), we rewrite them by using the lowenergy parameters for $Q=0$. Here, we neglected the $Q$-dependences of $\beta_{\lambda}$ and $\gamma_{M_{H}^{2}}$ as they are dominated by $Q$-independent interactions, i.e, the top Yukawa and the gauge interactions. 
Once the low energy parameters are given by eqs. (3.1) and (3.2), it is no longer possible to factor out the quintessence couplings from the Higgs potential. Thus, the Higgs VEV has a non-trivial dependence on the quintessence field; for $Q \ll M_{\mathrm{PL}}$, the Higgs VEV is obtained as

$$
\left.\langle H\rangle^{2} \simeq \frac{M_{H}^{2}\left(\mu_{R}\right)}{2 \lambda\left(\mu_{R}\right)}\right|_{Q=0} \times\left(1-\frac{c_{H} Q / M_{\mathrm{PL}}}{\left.\lambda\left(\mu_{R}\right)\right|_{Q=0}} \times\left.\int \frac{d \mu_{R}^{\prime}}{\mu_{R}^{\prime}} \beta_{\lambda}\left(\mu_{R}^{\prime}\right)\right|_{Q=0}\right) .
$$

Thus, the shift of the quintessence field induces the shift of the VEV. Numerically, we found

$$
\left.\frac{d \ln v_{H}(X)}{d X}\right|_{X=0} \simeq-0.5 \times c_{H}
$$

where $\lambda\left(m_{t}\right) \simeq 0.126$ and the integration of $\beta_{\lambda}$ is

$$
\int_{M_{\mathrm{PL}}}^{m_{t}} \frac{d \mu_{R}^{\prime}}{\mu_{R}^{\prime}} \beta_{\lambda}\left(\mu_{R}^{\prime}\right) \simeq 0.13
$$

obtained by using RGErun2. Here, we take the top quark mass, $m_{t}=173 \mathrm{GeV}$, as the low energy renormalization scale. ${ }^{8}$

Thus, from the constraints on the long range force in eq. (2.28), we again obtain an upper limit,

$$
\left|c_{H}\right| \lesssim 0.4 \times 10^{-4}
$$

while the tests of the time-variation of the proton-to-electron mass ratio in eq. (2.34) gives

$$
c_{Q}\left|c_{H}\right| \lesssim 0.4 \times 10^{-5}
$$

Therefore, we find that the Higgs potential $V_{H}^{(c)}$ is in tension with tests of the equivalence principle and the time-variation of the proton-to-electron mass ratio.

In the above argument, we have implicitly assumed that the quadratic and the quartic divergences appearing in the Higgs squared mass parameter and in the cosmological constant term are fine-tuned by local counter terms even if they have non-trivial dependences on the quintessence field. This means that the Higgs potential at high energy scale includes local terms of the form:

$$
V_{H}=V_{H}^{c}+\Lambda_{2}^{2}(X)|H|^{2}+\Lambda_{4}^{4}(X)
$$

in addition to $V_{H}^{(c)}$. Here, $\Lambda_{2}^{2}(X)$ and $\Lambda_{4}^{4}(X)$ are functions of the quintessence field which are introduced to cancel the $Q$-dependent quadratic and quartic divergences. These assumptions are justified by the anthropic principle, since otherwise, the vacuum is no more habitable [43-45].

We comment here that, as the local term $\Lambda_{2}^{2}(X)|H|^{2}$ is added to the high energy Lagrangian, eq. (3.1) does not hold in general. With such a term, there should exist a

\footnotetext{
${ }^{8}$ The relative error of the integration of eq. (3.4) is of $\mathcal{O}\left(10^{-1}\right)$ which is dominated by the choice of $\mu_{R}$. We will provide more precise discussion including the renormalization conditions in the next subsection.
} 
residual $Q$-dependent mass term due to the $\Lambda_{2}^{2}(X)|H|^{2}$ term which is not proportional to $e^{-c_{H} Q / M_{\mathrm{PL}}}$ in general. Such an observation does not weaken the constraints in eqs. (3.6) and (3.7).

As another comment, it is also tempting to ask whether it is possible to realize a phenomenologically viable scenario by assuming low energy parameters giving rise to the Higgs potential of the form of $V_{H}^{(c)}$ (see eq. (2.37)), i.e.,

$$
\begin{aligned}
M_{H}^{2}\left(\mu_{R} \simeq m_{t}\right) & \left.\simeq M_{H}^{2}\left(\mu_{R} \simeq m_{t}\right)\right|_{X=0} e^{-c_{H} Q / M_{\mathrm{PL}}}, \\
\lambda\left(\mu_{R} \simeq m_{t}\right) & \left.\simeq \lambda\left(\mu_{R} \simeq m_{t}\right)\right|_{X=0} e^{-c_{H} Q / M_{\mathrm{PL}}} .
\end{aligned}
$$

These renormalization conditions can be satisfied by adding local counter terms of the quartic coupling to $V_{H}^{(c)}$ at the high energy scale with appropriate quintessence field dependences. As we will see shortly, however, the Higgs VEV still depends on quintessence field even if the renormalization conditions in eqs. (3.9) and (3.10) are imposed. Let us also stress again that such fine-tuning conditions are not supported by the de Sitter swampland conjecture nor by the anthropic principle.

\subsection{Analysis in the $\overline{\mathrm{MS}}$ prescription}

To make our statement more precise and concrete, let us consider an effective field theory where the bare Higgs potential is given by

$$
V_{H B}\left(H_{B}\right)=-M_{H B}^{2}(X)\left|H_{B}\right|^{2}+\lambda_{B}(X)\left|H_{B}\right|^{4}+\Lambda_{E W B}^{4}(X),
$$

with the subscript $B$ denoting the bare parameters and fields. The $Q$-dependences of the coefficient parameter functions are not specified at this point. As for the other coupling constants in the Standard Model, we assume that they are independent of the quintessence field. We treat the quintessence field as a background field and do not consider the pathintegration of the quintessence field.

In this setup, the quantum effective potential of the Higgs boson is given by

$$
V_{H \mathrm{eff}}(H)=-M_{H}^{2(\overline{\mathrm{MS}})}(X)|H|^{2}+\lambda^{(\overline{\mathrm{MS}})}(X)|H|^{4}+\Lambda_{\mathrm{EW}}^{4(\overline{\mathrm{MS}})}(X)+V_{H \mathrm{eff}}^{(1)}+\cdots,
$$

where the first three terms are renormalized tree-level contributions while $V_{H \text { eff }}^{(n)}(n>0)$ are $n$-loop contributions. ${ }^{9}$ At the tree level, i.e., neglecting $V_{H \text { eff }}^{(n)}$ with $n>0$, the Higgs $\mathrm{VEV}$ is given by

$$
\left.v_{H}^{(\overline{\mathrm{MS}}) 2}(X)\right|_{\text {tree }}=\frac{M_{H}^{2(\overline{\mathrm{MS}})}(X)}{\lambda^{(\overline{\mathrm{MS}})}(X)} .
$$

In the following, we discuss how the Higgs VEV behaves after taking into account radiative corrections.

\footnotetext{
${ }^{9}$ In this paper, the quantum effective potential denotes the one calculated perturbatively for a quantum state whose wave functional is localized at around a particular field value. It differs from the one defined by the Legendre transformation of the partition function of the connected Green functions, $W[J][53]$.
} 
We adopt the $\overline{\mathrm{MS}}$ prescription for the renormalization for a given value of $X$. The one-loop effective potential $V_{H \text { eff }}^{(1)}$ is given by $[54,55]$

$$
\begin{aligned}
16 \pi^{2} V_{H \text { eff }}^{(1)}= & \frac{F_{H}^{2}}{4}\left(\overline{\ln } F_{H}-\frac{3}{2}\right)+\frac{3 F_{G}^{2}}{4}\left(\overline{\ln } F_{G}-\frac{3}{2}\right)-3 F_{T}^{2}\left(\overline{\ln } F_{T}-\frac{3}{2}\right) \\
& +\frac{3 F_{W}^{2}}{2}\left(\overline{\ln } F_{W}-\frac{5}{6}\right)+\frac{3 F_{Z}^{2}}{4}\left(\overline{\ln } F_{Z}-\frac{5}{6}\right),
\end{aligned}
$$

in the $\overline{\mathrm{MS}}$ prescription. The functions $F$ 's are given by

$$
\begin{aligned}
F_{H} & =-M_{H}^{2(\overline{\mathrm{MS}})}(X)+3 \lambda^{(\overline{\mathrm{MS}})}(X) h^{2}, \\
F_{G} & =-M_{H}^{2(\overline{\mathrm{MS}})}(X)+\lambda^{(\overline{\mathrm{MS}})}(X) h^{2} \\
F_{T} & =y_{t}^{(\overline{\mathrm{MS}}) 2} h^{2} / 2 \\
F_{W} & =g^{(\overline{\mathrm{MS}}) 2} h^{2} / 4 \\
F_{Z} & =\left(g^{(\overline{\mathrm{MS}}) 2}+g^{\prime(\overline{\mathrm{MS}}) 2}\right) h^{2} / 4 \\
\overline{\ln } F & =\ln F / \mu_{R}^{2}
\end{aligned}
$$

where $y_{t}, g$ and $g^{\prime}$ are the top Yukawa and the gauge coupling constants of the $\mathrm{SU}(2)_{L}$ and $\mathrm{U}(1)_{Y}$ gauge interactions in the $\overline{\mathrm{MS}}$ prescription, respectively. Here, we parametrize the Higgs doublet as follows without loss of generality,

$$
H=\frac{1}{\sqrt{2}}(0, h)^{T} .
$$

Following refs. [54, 55], we define the $\overline{\mathrm{MS}} \mathrm{VEV}, v_{H}^{(\overline{\mathrm{MS}})}(X)$, by the field value of $h$ which minimizes $V_{H \text { eff }}$ in the $\overline{\mathrm{MS}}$ prescription. Then, the $\overline{\mathrm{MS}}$ Higgs VEV does not satisfy the tree-level relation given in eq. (3.13). As the VEV of the renormalized Higgs field is not a physical observable, its definition beyond the tree level is arbitrary and a matter of convention. The advantage of the definition in refs. $[54,55]$ is that the Higgs tadpole diagrams are cancelled by definition. With the present definition of $v_{H}^{(\overline{\mathrm{MS}})}(X)$, the pole electron mass is given by

$$
m_{e}(X)=\frac{1}{\sqrt{2}} y_{e}^{(\overline{\mathrm{MS}})} v_{H}^{(\overline{\mathrm{MS}})}(X) \times\left(1-\Re \Sigma_{S}\left(m_{e}^{2}\right)-\Re \Sigma_{V}\left(m_{e}^{2}\right)\right),
$$

where $\Sigma_{V, S}\left(p^{2}\right)$ are defined by the free electron self-energy, $\Sigma_{e}$,

$$
\Sigma_{e}(p)=i p \Sigma_{V}\left(p^{2}\right)+i m_{e} \Sigma_{S}\left(p^{2}\right),
$$

at one loop. ${ }^{10}$ As the Higgs tadpole diagrams automatically vanish in $\Sigma_{e}$, the $Q$ dependence of $m_{e}(X)$ is dominated by the one through $v_{H}^{(\overline{\mathrm{MS}})}(X)$. The $Q$-dependence

\footnotetext{
${ }^{10}$ Since the electron mass is much smaller than those of $Z$ and $W$ bosons appearing in $\Sigma_{e}$, it is not practical to calculate $m_{e}$ by using $\Sigma_{e}$ obtained in the Standard Model. Rather, we need to match the $\overline{\mathrm{MS}}$ electron masses in the Standard Model and in the low energy effective theory below the electroweak scale. Those procedures do not affect our argument, though.
} 
though $M_{H}^{2(\overline{\mathrm{MS}})}(X)$ comes from the Higgs-electron loop contribution to $\Sigma_{S, V}\left(m_{e}^{2}\right)$, which is proportional to the electron Yukawa coupling squared, and is numerically unimportant. As a result, we find,

$$
\left.\left.\frac{d \ln m_{e}(X)}{d X}\right|_{X=0} \simeq \frac{d \ln v_{H}^{(\overline{\mathrm{MS}})}(X)}{d X}\right|_{X=0}
$$

Similarly, the quark masses also depend on the quintessence field via $v_{H}^{(\overline{\mathrm{MS}})}$, and we find

$$
\left.\left.\frac{d \ln m_{N}(X)}{d X}\right|_{X=0} \simeq f_{N} \frac{d \ln v_{H}^{(\overline{\mathrm{MS}})}(X)}{d X}\right|_{X=0}
$$

It should be noted that the quintessence field couples to the top quark not only through $v_{H}^{(\overline{\mathrm{MS}})}(X)$ but also through radiative corrections in which the Higgs boson is circulating. For now, we neglect these couplings and we will come back to this point later.

Now, let us discuss renormalization conditions. From a perspective of low energy effective field theory, we only know the Higgs potential parameters in the present universe, i.e. $X=0$. For $X \neq 0$, there is no experimental data to determine the renormalization conditions. Thus, we may, for example, impose $X$-dependences of the parameter functions of $X$, so that

$$
\begin{aligned}
M_{H}^{2(\overline{\mathrm{MS}})}(X) & =M_{H}^{2(\overline{\mathrm{MS}})}(X=0) e^{-c_{H} X}, \\
\lambda^{(\overline{\mathrm{MS}})}(X) & =\lambda^{(\overline{\mathrm{MS}})}(X=0) e^{-c_{H} X},
\end{aligned}
$$

at a low energy scale such as the pole mass of the top quark, $\mu_{R}=m_{t}^{(\text {pole })}$. These conditions correspond to the ones in eqs. (3.9) and (3.10) in our argument in the Wilsonian approach. With these conditions, the tree-level Higgs VEV, $\left.v_{H}^{(\overline{\mathrm{MS}})}\right|_{\text {tree }}$, is independent of the quintessence field. We call these renormalization conditions as the low energy quintessence (LQ) prescription. It should be stressed here that conspiratorial fine-tunings are hidden in the renormalization conditions in eqs. (3.26) and (3.27) from a perspective of the highenergy theory.

Beyond the tree level, the Higgs VEV does not satisfy the tree-level relation. At the one-loop level, for example, the shift of the VEV is roughly given by

$$
\frac{v_{H}^{(\overline{\mathrm{MS}})}(X)-\left.v_{H}^{(\overline{\mathrm{MS}})}\right|_{\text {tree }}}{\left.v_{H}^{(\overline{\mathrm{MS}})}\right|_{\text {tree }}} \simeq \frac{1}{\left.2 \lambda^{(\overline{\mathrm{MS}})}(X) v_{H}^{(\overline{\mathrm{MS}}) 3}\right|_{\text {tree }}} \frac{\partial V^{(1)}}{\partial h} \sim-\frac{9}{64 \pi^{2} \lambda^{(\overline{\mathrm{MS}})}(X)} y_{t}^{(\overline{\mathrm{MS}}) 4},
$$

where we keep only the top Yukawa contribution in eq. (3.14) for presentation purpose. Thus, the deviation of the Higgs VEV from the tree-level relation in eq. (3.13) results in a non-trivial quintessence field dependence, which is enhanced by $1 / \lambda^{(\overline{\mathrm{MS}})}$.

Our numerical analysis is as follows. We first calculate $v_{H}^{(\overline{\mathrm{MS}})}(X=0)$ at $\mu_{R}=m_{t}^{(\text {pole })}$ from the relation between $v_{H}^{(\overline{\mathrm{MS}})}(X=0)$ and the Fermi coupling constant $G_{\mu}$ in [56], which 
leads to ${ }^{11}$

$$
v_{H}^{(\overline{\mathrm{MS}})}(X=0)=246.8 \mathrm{GeV} .
$$

Then, we obtain the quartic coupling and the Higgs mass parameters at $X=0$,

$$
\begin{aligned}
\lambda_{H}^{(\overline{\mathrm{MS}})}(X=0) & =0.1261 \pm 0.0003, \\
M_{H}^{2(\overline{\mathrm{MS}})}(X=0) & =(92.9 \pm 0.1 \mathrm{GeV})^{2},
\end{aligned}
$$

at $\mu_{R}=m_{t}^{\text {(pole) }}$. In evaluating these values, we use SMH [58], which takes full two-loop and leading three-loop corrections into account. ${ }^{12}$ The uncertainties quoted here do not include the ones from the choice of the renormalization scale. The input parameters for SMH are taken to be

$$
\begin{aligned}
m_{Z}^{(\text {pole })} & =91.1876 \pm 0.0021 \mathrm{GeV}, \\
m_{W}^{(\text {pole })} & =80.379 \pm 0.012 \mathrm{GeV}, \\
m_{h}^{(\text {pole })} & =125.18 \pm 0.16 \mathrm{GeV} \\
m_{t}^{(\text {pole })} & =173.0 \pm 0.4 \mathrm{GeV} \\
\alpha_{s}^{(\overline{\mathrm{MS}})}\left(m_{z}\right) & =0.1181 \pm 0.0011
\end{aligned}
$$

(see ref. [57]). The uncertainties of the parameters in eqs. (3.30) and (3.31) are dominated by the uncertainty of $m_{h}^{(\mathrm{pole})}$. The values $v_{H}^{(\overline{\mathrm{MS}})}(X=0), \lambda_{H}^{(\overline{\mathrm{MS}})}(X=0)$, and $M_{H}^{2(\overline{\mathrm{MS}})}(X=0)$ are taken as reference values to estimate the shift of the Higgs VEV for $X \neq 0$. As we are interested in $d \ln v_{H}^{(\overline{\mathrm{MS}})} / d X$, the uncertainties of those parameters are cancelled at the leading order.

In figure 1 , we show how $v_{H}^{(\overline{\mathrm{MS}})}$ shifts in the LQ prescription, where $\lambda^{(\overline{\mathrm{MS}})}$ and $M_{H}^{2(\overline{\mathrm{MS}})}$ are changed while $M_{H}^{2(\overline{\mathrm{MS}})} / \lambda^{\overline{\mathrm{MS}}}$ is fixed. Here, we again utilize SMH [58] to obtain $v_{H}^{(\overline{\mathrm{MS}})}(X \neq$ $0)$ for given $\lambda^{(\overline{\mathrm{MS}})}(X \neq 0)$ and $M_{H}^{2(\overline{\mathrm{MS}})}(X \neq 0)$. The figure shows that, based on a calculation including the leading three-loop effects, the $\overline{\mathrm{MS}}$ Higgs VEV changes as ${ }^{13}$

$$
\frac{\Delta v_{H}^{(\overline{\mathrm{MS}})}}{v_{H}^{(\overline{\mathrm{MS}})}} \simeq 0.07 \times \frac{\Delta \lambda^{(\overline{\mathrm{MS}})}}{\lambda^{(\overline{\mathrm{MS}})}} .
$$

The rather large $X$ dependence in eq. (3.37) stems from the fact that the shift of the VEV from the tree-level relation is enhanced by $1 / \lambda^{(\overline{\mathrm{MS}})}$ (see eq. (3.28)). Combining with the renormalization conditions of the LQ prescription (eqs. (3.26) and (3.27)), we find

$$
\left.\frac{d \ln v_{H}^{(\overline{\mathrm{MS}})}(X)}{d X}\right|_{X=0} \simeq-0.07 \times c_{H} .
$$

\footnotetext{
${ }^{11}$ A nominal uncertainty, $v_{H}^{(\overline{\mathrm{MS}})}(X=0)=246.7711 \pm 0.0015 \mathrm{GeV}\left(\mu_{R}=m_{\mathrm{top}}^{(\text {pole })}\right)$, is dominated by the error of the top quark mass, $m_{t}^{(\text {pole })}=173.0 \pm 0.4 \mathrm{GeV}[57]$, although we do not need a very precise value of it.

${ }^{12}$ In SMH, the loop integrations are handled by TSIL [59].

${ }^{13}$ We choose the renormalization scale to be $\mu_{R}=m_{\mathrm{t}}^{\text {(pole) }}$ even for $X \neq 0$.
} 


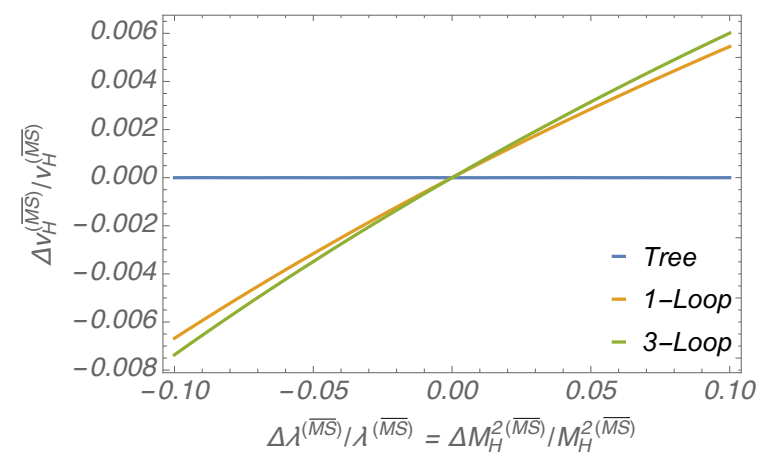

Figure 1. The shift of the vacuum expectation value of the Higgs VEV in the LQ prescription in eq. (3.26) and (3.27), where SMH is used for a numerical calculation at tree, one-loop, and three-loop level. The figure shows that the Higgs VEV does not shift at the tree-level due to the tree level relation of the Higgs VEV in eq. (3.13). Beyond the tree-level, the Higgs VEV is shifted by changing $\lambda^{(\overline{\mathrm{MS}})}$.

As a result, we find that the tests of the equivalence principle and the time-variation of the proton-to-electron mass ratio leads to slightly weakened conditions,

$$
c_{H} \lesssim 0.3 \times 10^{-3}
$$

and

$$
c_{Q}\left|c_{H}\right| \lesssim 0.3 \times 10^{-4}
$$

respectively. ${ }^{14}$ Thus, the Higgs potential $V_{H}^{(c)}$ with $\mathcal{O}(1)$ coefficients is in tension with the observational constraints even in the LQ prescription.

So far, we have imposed the renormalization conditions (3.26) and (3.27) at the electroweak scale without assuming any particular high energy theory. As another possibility, we may impose them at $\mu_{R} \simeq M_{\mathrm{PL}}$. This possibility corresponds to the case discussed in the previous subsection, i.e., the case with the Wilsonian effective action with $V_{H}^{(c)}$ at around the Planck scale. We call these renormalization conditions as the high energy $\overline{\mathrm{MS}}$ quintessence $(\mathrm{HQ})$ prescription. In this case, $d \ln v_{H}^{(\overline{\mathrm{MS}})}(X) /\left.d X\right|_{X=0} \simeq-0.5 \times c_{H}$ (see eq. (3.4)), which is an order of magnitude larger than the LQ case. Therefore, we again conclude that the HQ prescription defined in the $\overline{\mathrm{MS}}$ scheme is in a strong tension with the current constraints (see eqs. (3.6) and (3.7)).

Several comments are in order. In the LQ prescription, the reason why $v_{H}^{(\overline{\mathrm{MS}})}$ shifts is that the effective Higgs potential is modified by the $n$-loop contributions, $V_{H \text { eff }}^{(n)}$. At the one-loop level, for example, the most relevant terms for the VEV shift are the second terms of each contribution in eq. (3.14). As they are proportional to the tree-level terms in eq. (3.12), we can eliminate the effects of those times for $X \neq 0$ by carefully adjusting

\footnotetext{
${ }^{14}$ Recently, it has been argued that the change of the Higgs quartic coupling by about a ten percent level at low energy due to the quintessence field may stabilize the Higgs vacuum in the Standard Model [33]. Our result shows that such a possibility has a tension with the constraint in eq. (2.34).
} 
the renormalization conditions such that,

$$
\begin{aligned}
M_{H}^{2(\overline{\mathrm{MS}})}(X)= & M_{H}^{2(\overline{\mathrm{MS}})}(X=0) e^{-c_{H} X}+\left(1-e^{-c_{H} X}\right) \frac{9}{16 \pi^{2}} \lambda^{(\overline{\mathrm{MS}})} m_{H}^{2(\overline{\mathrm{MS}})}+\cdots \\
\lambda^{(\overline{\mathrm{MS}})}(X)= & \lambda^{(\overline{\mathrm{MS}})}(X=0) e^{-c_{H} X} \\
& -\left(1-e^{-c_{H} X}\right)\left(\frac{9}{32 \pi^{2}} y_{t}^{(\overline{\mathrm{MS}}) 4}-\frac{15}{512 \pi^{2}} g^{(\overline{\mathrm{MS}}) 4}-\frac{5}{256 \pi^{2}} g^{(\overline{\mathrm{MS}}) 2} g^{\prime(\overline{\mathrm{MS}}) 2}\right. \\
& \left.-\frac{5}{512 \pi^{2}} g^{\prime(\overline{\mathrm{MS}}) 2} g^{\prime(\overline{\mathrm{MS}}) 2}-\frac{9}{8 \pi^{2}} \lambda^{(\overline{\mathrm{MS}}) 2}(X=0)\right)+\cdots
\end{aligned}
$$

Here, the ellipses denote the terms required to cancel the $Q$-dependence of the Higgs VEV through higher order contributions. As we repeatedly argued in this paper, such an additional requirements are not justified by the de Sitter swampland conjecture nor by the anthropic principle, though.

In eq. (3.14), the terms which logarithmically depend on $|H|^{2}$, on the other hand, cannot be eliminated by local counter terms. Thus, even the meticulously-tuned renormalization conditions in eqs. (3.41) and (3.42) do not cancel the $Q$-dependence of the Higgs VEV completely. Numerically, we find that these renormalization conditions lead to

$$
\left.\frac{d \ln v_{H}^{(\overline{\mathrm{MS}})}}{d X}\right|_{X=0} \simeq 0.0037 \times c_{H},
$$

at the one-loop level. Correspondingly, the constraints on the long-range force and the time-varying proton-to-electron mass ratio lead to

$$
c_{H} \lesssim 0.6 \times 10^{-2},
$$

and

$$
c_{Q}\left|c_{H}\right| \lesssim 0.5 \times 10^{-3}
$$

Therefore, even highly conspiratorial renormalization conditions are still in tension with the de Sitter swampland conjecture.

\subsection{Another constraint}

So far, we have discussed constraints which are in association with the $Q$-dependence of the Higgs VEV. Here, we comment on another constraint. It is less severe compared to the previous ones if the Higgs VEV has $Q$-dependence, but is applicable even if the Higgs VEV is independent of $Q$ as we show in the following. (The constraint in this section is also studied in [29].)

In the model of our interest, the coupling of $Q$ to the top quark is radiatively generated as we have mentioned earlier. In figure 2, we show the Feynman diagram generating the $Q \bar{t} t$ vertex, where the trilinear scalar interaction shows up by expanding the scalar potential around $h \simeq v_{H}$. As a result, the $Q \bar{t} t$ vertex is given as ${ }^{15}$

$$
\mathcal{L}_{\mathrm{eff}} \simeq-\frac{1}{32 \pi^{2}} y_{t}^{(\overline{\mathrm{MS}}) 2} I\left(M_{t}^{(\overline{\mathrm{MS}}) 2} / 2 M_{H}^{2(\overline{\mathrm{MS}})}\right) \frac{M_{t}^{(\overline{\mathrm{MS}})}}{M_{\mathrm{PL}}} c_{H} Q \bar{t} t \simeq-0.0015 \times \frac{M_{t}^{(\overline{\mathrm{MS}})}}{M_{\mathrm{PL}}} c_{H} Q \bar{t} t
$$

\footnotetext{
${ }^{15}$ Here, we use the tree-level relation, $M_{t}^{(\overline{\mathrm{MS}})}=y_{t}^{(\overline{\mathrm{MS}})} v_{H}^{(\overline{\mathrm{MS}})} / \sqrt{2}$, which is enough at the one-loop level.
} 


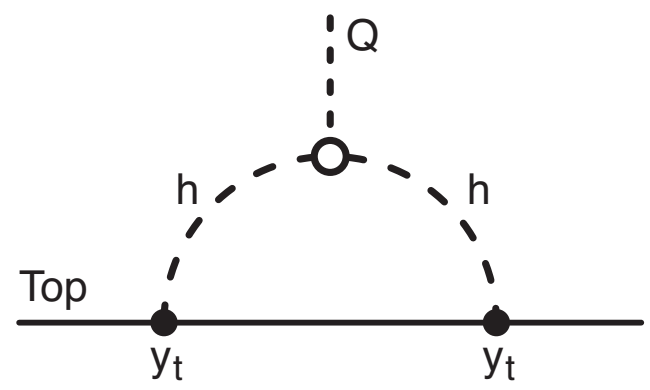

Figure 2. Feynman diagram which radiatively generates $Q \bar{t} t$ vertex. Here, $y_{t}$ denotes the top-quark Yukawa coupling constant.

where

$$
I(x)=\frac{1-x+x \log x}{(1-x)^{2}} .
$$

Here again, we assume $M_{H}^{2(\overline{\mathrm{MS}})}(X) \propto e^{-c_{H} X}$, while the top Yukawa coupling $y_{t}^{(\overline{\mathrm{MS}})}$ is independent of $X$ as in the case of the LQ prescription. The coupling of $Q$ to the lighter fermions are suppressed by the Yukawa coupling, and hence, less important.

The effective vertex in eq. (3.46) eventually leads to the coupling to the nucleons,

$$
\begin{aligned}
\mathcal{L}_{\text {eff }} & \simeq-\frac{2}{27}\left(1-\sum_{q=u, d, s} f_{q}^{N}\right) \times 0.0015 \times \frac{m_{N}}{M_{\mathrm{PL}}} c_{H} Q \bar{\psi}_{N} \psi_{N} \\
& \simeq 1 \times 10^{-4} \times \frac{m_{N}}{M_{\mathrm{PL}}} c_{H} Q \bar{\psi}_{N} \psi_{N} .
\end{aligned}
$$

Thus, the tests on the long range force in eq. (2.28) put a constraint,

$$
\left|c_{H}\right| \lesssim 0.08
$$

while the tests of the time-variation of the proton-to-electron mass ratio in eq. (2.34) lead to

$$
c_{Q}\left|c_{H}\right| \lesssim 2 \times 10^{-2}
$$

Those constraints are independent of the ones derived from the $Q$-dependence of the Higgs VEV.

\section{Conclusions and discussions}

The recently proposed de Sitter swampland conjecture excludes local extrema of a scalar potential with a positive energy density in a low energy effective theory. Combining with the habitable conditions of the vacua in the string landscape, the Higgs potential is required to be retrofitted to have non-trivial couplings to the quintessence field $Q$ so that the vacuum energy stays very low in the course of cosmological evolution.

In this paper, we found that the retrofitted Higgs potential generically predicts that the Higgs VEV becomes dependent on the amplitude of the quintessence field. We first 
discussed that the Higgs VEV shows a sizable $Q$-dependence based on the general Higgs potential (see e.g. $V_{H}^{(b)}$ in eq. (2.12)), which is consistent with the de Sitter swampland conjecture as well as the anthropic principle. We also argued that the overall coupling of the quintessence filed to the Higgs potential at a high energy scale (see e.g. $V_{H}^{(c)}$ in eq. (2.37)) results in a $Q$-dependent Higgs VEV due to the renormalization-group runnings. Furthermore, we also found that, even if $Q$ has the overall coupling to the Higgs potential at a low energy scale, the Higgs VEV is still $Q$-dependent. Those conclusions do not depend on the details of the quintessence-Higgs couplings nor the potential of the quintessence field as long as they satisfy the de Sitter swampland conjecture and the anthropic principle. As a result, we conclude that most of the habitable vacua with a Higgs potential which satisfies the de Sitter swampland conjecture predicts sizable $Q$-dependence of the Higgs VEV unless there is a fundamental reason to exclude a $Q$-dependent Higgs expectation value. As we have discussed, the scenario with the $Q$-dependent Higgs VEV contradicts with the tests of the equivalence principle as well as the tests of the time-varying proton-to-electron mass ratio.

Similarly, if there exists a scalar field which provides masses to colored particles, then the $Q$-dependence of its VEV is required to be weak enough to avoid the constraints from the long-range force and the time-varying proton-to-electron mass ratio. The examples of such scalar fields include the field which breaks the Peccei-Quinn symmetry or the Grand Unified gauge symmetry.

In summary, if a quintessence field $Q$ is coupled to the Higgs potential (as well as to dark energy) to satisfy the swampland conjecture, the scenario is severely constrained by the long-range force and the time-dependence of the proton-to-electron mass ratio. Unless there exists any additional principle to avoid these constraints, it seems difficult to find ourselves living in a vacuum consistent with phenomenological constraints.

Note added. After this paper was submitted, a refined version of the de Sitter swampland conjecture is proposed [60], which evades the problems discussed in this paper. In this paper, we are concerned with the original proposal of [4] which turns out to be severely constrained.

\section{Acknowledgments}

The authors would like to thank M. Yamazaki and T. T. Yanagida for valuable discussion. The authors also thank K. Choi, D. Chway and C. S. Shin for sharing their analysis on the constraints on the radiatively generated $Q$-nucleon coupling. This work is supported in part by Grants-in-Aid for Scientific Research from the Ministry of Education, Culture, Sports, Science, and Technology (MEXT) KAKENHI, Japan, No. 15H05889, No. 16H03991, No. 17H02878, and No.18H05542 (M.I.), No.16H02189, No.26104001, and No. 26104009 (K.H.), No. 16H06490, and No. 18K03608 (T.M.), and by the World Premier International Research Center Initiative (WPI), MEXT, Japan. 


\section{A Time evolution of quintessence field}

In this appendix, we briefly discuss how the (real-valued) quintessence field, $Q$, evolves from the time of the redshift parameter $z=\mathcal{O}(1)$, to the present. (For more detailed analysis of the evolution of the quintessence field with the potential in eq. (2.1), see e.g. refs. [39, 61, 62].) For $z<\mathcal{O}(10)$, the Hubble parameter is well approximated by

$$
H=H_{0} \sqrt{\frac{\Omega_{m}}{a^{3}}+\frac{1}{3 H_{0}^{2} M_{\mathrm{PL}}^{2}}\left(\frac{1}{2} \dot{Q}^{2}+V_{Q}(Q)\right)},
$$

where the dot denotes the time derivative, and $a$ the scale factor of the universe. The first term in the right-hand side denotes the contribution of the non-relativistic matter (with $\Omega_{m} \simeq 0.3$ being the density parameter of matter [42]), and the second term the one of the quintessence field $Q$ which plays a role of the dark energy. All the other scalar fields than the quintessence field have settled to their VEVs well before $z \simeq \mathcal{O}(1)$.

To demonstrate how $Q$ evolves, we consider the simplest potential of the quintessence field in eq. (2.1). In the slow-rolling regime, $c_{Q} \ll \sqrt{6}$, the energy density of the quintessence field is dominated by the potential energy. The equation of motion of $Q$ is given by

$$
\ddot{Q}+3 H \dot{Q}=-\partial V_{Q} / \partial Q=3 \xi_{c c} H_{0}^{2} M_{\mathrm{PL}} c_{Q} e^{-c_{Q} Q / M_{\mathrm{PL}}} .
$$

By using rescaled variables,

$$
X=\frac{Q}{M_{\mathrm{PL}}}, \quad x=\sqrt{3} H_{0} t, \quad X^{\prime}=\frac{d X}{d x}=\frac{1}{\sqrt{3} H_{0} M_{\mathrm{PL}}} \frac{d Q}{d t}, \quad \tilde{V}_{Q}(X)=\xi_{\mathrm{cc}} e^{-c_{Q} X},
$$

the Hubble equation and the equation of motion of $Q$ are reduced to

$$
\begin{aligned}
\frac{a^{\prime}}{a} & =\frac{1}{\sqrt{3}} \sqrt{\frac{\Omega_{m}}{a^{3}}+\frac{1}{2} X^{\prime 2}+\tilde{V}_{Q}(X)}, \\
X^{\prime \prime} & +\sqrt{3} \sqrt{\frac{\Omega_{m}}{a^{3}}+\frac{1}{2} X^{\prime 2}+\tilde{V}_{Q}(X)} X^{\prime}=\xi_{c c} c_{Q} e^{-c_{Q} X} .
\end{aligned}
$$

We solve these equations with the boundary conditions,

$$
\begin{aligned}
a\left(t_{0}\right) & =1, \\
X\left(t_{0}\right) & =0, \\
\frac{1}{2} X^{\prime 2}+\left.\tilde{V}_{Q}(X)\right|_{t=t_{0}} & =0.7,
\end{aligned}
$$

with $t_{0}$ being the present cosmic time. The initial condition of $X^{\prime}$ is taken so that the motion of the quintessence is determined by the Hubble friction in the matter dominated era.

In figure 3, we show the evolution of the quintessence field as a function of the redshift parameter $z$ (the left panel). The figure shows that the field excursion from $z=1$ to $z=0$ is

$$
\left.\frac{\Delta Q}{M_{\mathrm{PL}}}\right|_{z=1} \simeq-0.24 \times c_{Q},
$$



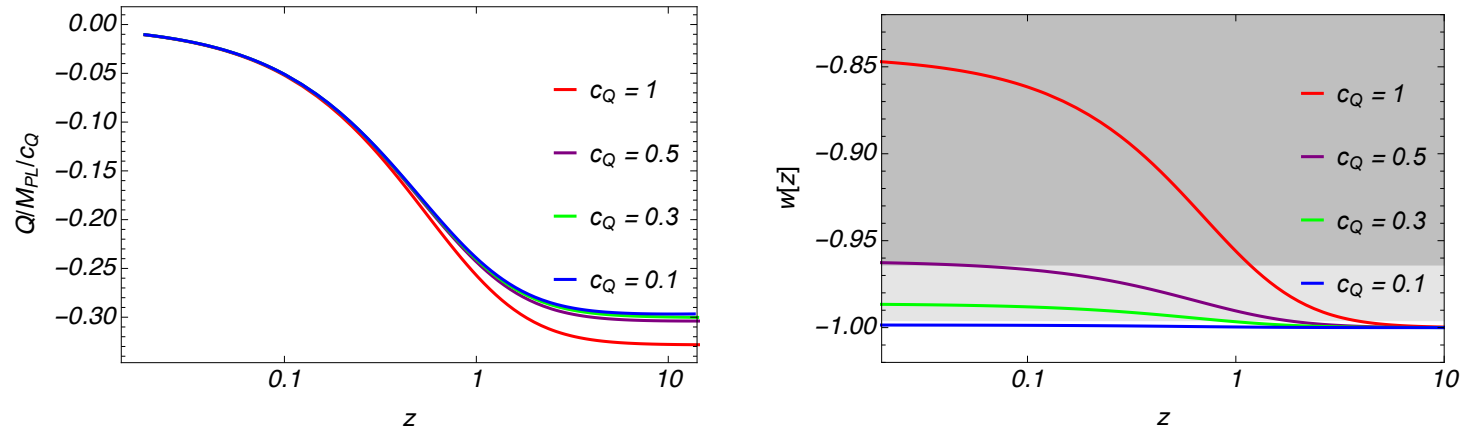

Figure 3. (Left) The excursion of the quintessence field from $Q(z=0)=0$ (normalized by $\left.M_{\mathrm{PL}} c_{Q}\right)$ as a function of the redshift parameter $z$ for $c_{Q}=1,0.5,0.3$, and 0.1. (Right) The equation of state of the dark energy as a function of the redshift parameter. The (light-)gray shaded region is disfavored by the $2 \sigma(1 \sigma)$ limit based on $\mathrm{CMB}$, SNe and BAO measurements [42], $w=-1.028 \pm 0.032(1 \sigma)$. Here, the energy density of the present universe are taken to be $\Omega_{m}=0.3$ and $\Omega_{\mathrm{DE}}=0.7$.

for $c_{Q} \lesssim 0.5$. In the right panel, we also show the equation of state of the dark energy,

$$
w=-1+\frac{\dot{Q}^{2}}{\frac{1}{2} \dot{Q}^{2}+V_{Q}(Q)} .
$$

The equation of state is larger than -1 as the quintessence field is in motion. The (light)gray shaded region is disfavored by the $2 \sigma(1 \sigma)$ limit from $\mathrm{CMB}, \mathrm{SNe}$ and $\mathrm{BAO}$ measurements [42], i.e. $w=-1.028 \pm 0.032(1 \sigma) .{ }^{16}$ The figure shows that $c_{Q}=1$ is excluded while $c_{Q}<0.5$ is within the allowed region.

Open Access. This article is distributed under the terms of the Creative Commons Attribution License (CC-BY 4.0), which permits any use, distribution and reproduction in any medium, provided the original author(s) and source are credited.

\section{References}

[1] C. Vafa, The string landscape and the swampland, hep-th/0509212 [INSPIRE].

[2] H. Ooguri and C. Vafa, On the geometry of the string landscape and the swampland, Nucl. Phys. B 766 (2007) 21 [hep-th/0605264] [INSPIRE].

[3] H. Ooguri and C. Vafa, Non-supersymmetric AdS and the swampland, Adv. Theor. Math. Phys. 21 (2017) 1787 [arXiv:1610.01533] [INSPIRE].

[4] G. Obied, H. Ooguri, L. Spodyneiko and C. Vafa, De Sitter space and the swampland, arXiv: 1806.08362 [INSPIRE].

[5] S. Vagnozzi et al., Constraints on the sum of the neutrino masses in dynamical dark energy models with $w(z) \geq-1$ are tighter than those obtained in $\Lambda C D M$, Phys. Rev. D 98 (2018) 083501 [arXiv: 1801.08553] [INSPIRE].

\footnotetext{
${ }^{16}$ This constraint is obtained by assuming that $w$ is constant in time. For more detailed analysis with the evolution of $w$ is taken into account, see e.g. ref. [6].
} 
[6] P. Agrawal, G. Obied, P.J. Steinhardt and C. Vafa, On the cosmological implications of the string swampland, Phys. Lett. B 784 (2018) 271 [arXiv:1806.09718] [INSPIRE].

[7] D. Andriot, On the de Sitter swampland criterion, Phys. Lett. B 785 (2018) 570 [arXiv: 1806.10999] [INSPIRE].

[8] A. Achúcarro and G.A. Palma, The string swampland constraints require multi-field inflation, arXiv: 1807.04390 [INSPIRE].

[9] S.K. Garg and C. Krishnan, Bounds on slow roll and the de Sitter swampland, arXiv: 1807.05193 [INSPIRE].

[10] J.-L. Lehners, Small-field and scale-free: inflation and Ekpyrosis at their extremes, JCAP 11 (2018) 001 [arXiv: 1807.05240] [INSPIRE].

[11] A. Kehagias and A. Riotto, A note on inflation and the swampland, arXiv:1807.05445 [INSPIRE].

[12] M. Dias, J. Frazer, A. Retolaza and A. Westphal, Primordial gravitational waves and the swampland, arXiv:1807.06579 [INSPIRE].

[13] F. Denef, A. Hebecker and T. Wrase, De Sitter swampland conjecture and the Higgs potential, Phys. Rev. D 98 (2018) 086004 [arXiv: 1807.06581] [INSPIRE].

[14] E.Ó. Colgáin, M.H. P.M. Van Putten and H. Yavartanoo, Observational consequences of $H_{0}$ tension in de Sitter Swampland, arXiv:1807.07451 [INSPIRE].

[15] R. Brandenberger, L.L. Graef, G. Marozzi and G.P. Vacca, Back-reaction of super-hubble cosmological perturbations beyond perturbation theory, arXiv:1807.07494 [INSPIRE].

[16] S. Paban and R. Rosati, Inflation in multi-field modified DBM potentials, JCAP 09 (2018) 042 [arXiv: 1807.07654] [INSPIRE].

[17] A. Ghalee, Condensation of a scalar field non-minimally coupled to gravity in a cosmological context, arXiv: 1807.08620 [INSPIRE].

[18] H. Matsui and F. Takahashi, Eternal inflation and swampland conjectures, arXiv: 1807.11938 [INSPIRE].

[19] I. Ben-Dayan, Draining the swampland, arXiv:1808.01615 [INSPIRE].

[20] C.-I. Chiang and H. Murayama, Building supergravity quintessence model, arXiv: 1808.02279 [INSPIRE].

[21] L. Heisenberg, M. Bartelmann, R. Brandenberger and A. Refregier, Dark energy in the swampland, arXiv: 1808.02877 [INSPIRE].

[22] C. Damian and O. Loaiza-Brito, Two-field axion inflation and the swampland constraint in the flux-scaling scenario, arXiv:1808.03397 [INSPIRE].

[23] W.H. Kinney, S. Vagnozzi and L. Visinelli, The zoo plot meets the swampland: mutual (in) consistency of single-field inflation, string conjectures and cosmological data, arXiv: 1808.06424 [INSPIRE].

[24] Y. Akrami, R. Kallosh, A. Linde and V. Vardanyan, The landscape, the swampland and the era of precision cosmology, arXiv:1808.09440 [INSPIRE].

[25] L. Heisenberg, M. Bartelmann, R. Brandenberger and A. Refregier, Dark energy in the swampland II, arXiv:1809.00154 [INSPIRE]. 
[26] H. Murayama, M. Yamazaki and T.T. Yanagida, Do we live in the swampland?, arXiv: 1809.00478 [INSPIRE].

[27] M.C.D. Marsh, The swampland, quintessence and the vacuum energy, arXiv:1809.00726 [INSPIRE].

[28] S. Brahma and M. Wali Hossain, Avoiding the string swampland in single-field inflation: excited initial states, arXiv: 1809.01277 [INSPIRE].

[29] K. Choi, D. Chway and C.S. Shin, The dS swampland conjecture with the electroweak symmetry and QCD chiral symmetry breaking, arXiv:1809.01475 [INSPIRE].

[30] S. Das, A note on single-field inflation and the swampland criteria, arXiv:1809.03962 [INSPIRE].

[31] U. Danielsson, The quantum swampland, arXiv:1809.04512 [INSPIRE].

[32] R.H. Brandenberger, Beyond standard inflationary cosmology, arXiv:1809.04926 [INSPIRE].

[33] C. Han, S. Pi and M. Sasaki, Quintessence saves Higgs instability, arXiv: 1809.05507 [INSPIRE].

[34] R. Brandenberger, R.R. Cuzinatto, J. Fröhlich and R. Namba, New scalar field quartessence, arXiv: 1809.07409 [INSPIRE].

[35] H. Matsui, F. Takahashi and M. Yamada, Isocurvature perturbations of dark energy and dark matter from the swampland conjecture, arXiv:1809.07286 [INSPIRE].

[36] M. Cicoli et al., De Sitter vs. quintessence in string theory, Fortsch. Phys. (2018) 1800079 [arXiv: 1808.08967] [INSPIRE].

[37] B. Ratra and P.J.E. Peebles, Cosmological consequences of a rolling homogeneous scalar field, Phys. Rev. D 37 (1988) 3406 [INSPIRE].

[38] C. Wetterich, Cosmology and the fate of dilatation symmetry, Nucl. Phys. B 302 (1988) 668 [arXiv: 1711.03844] [INSPIRE].

[39] S. Tsujikawa, Quintessence: a review, Class. Quant. Grav. 30 (2013) 214003 [arXiv: 1304.1961] [INSPIRE].

[40] T.A. Wagner, S. Schlamminger, J.H. Gundlach and E.G. Adelberger, Torsion-balance tests of the weak equivalence principle, Class. Quant. Grav. 29 (2012) 184002 [arXiv:1207.2442] [INSPIRE].

[41] C.J. A.P. Martins, The status of varying constants: a review of the physics, searches and implications, arXiv:1709.02923 [INSPIRE].

[42] Planck collaboration, N. Aghanim et al., Planck 2018 results. VI. Cosmological parameters, arXiv: 1807.06209 [INSPIRE].

[43] R. Bousso and J. Polchinski, Quantization of four form fluxes and dynamical neutralization of the cosmological constant, JHEP 06 (2000) 006 [hep-th/0004134] [INSPIRE].

[44] L. Susskind, The anthropic landscape of string theory, hep-th/0302219 [INSPIRE].

[45] M. Tegmark, A. Aguirre, M. Rees and F. Wilczek, Dimensionless constants, cosmology and other dark matters, Phys. Rev. D 73 (2006) 023505 [astro-ph/0511774] [INSPIRE].

[46] M.A. Shifman, A.I. Vainshtein and V.I. Zakharov, Remarks on Higgs boson interactions with nucleons, Phys. Lett. B 78 (1978) 443. 
[47] M. Hoferichter, P. Klos, J. Menéndez and A. Schwenk, Improved limits for Higgs-portal dark matter from LHC searches, Phys. Rev. Lett. 119 (2017) 181803 [arXiv:1708.02245] [INSPIRE].

[48] A. Crivellin, M. Hoferichter and M. Procura, Accurate evaluation of hadronic uncertainties in spin-independent WIMP-nucleon scattering: Disentangling two- and three-flavor effects, Phys. Rev. D 89 (2014) 054021 [arXiv:1312.4951] [InSPIRE].

[49] D. Wang, The multi-feature universe: large parameter space cosmology and the swampland, arXiv: 1809.04854 [INSPIRE].

[50] L.J. Hall, D. Pinner and J.T. Ruderman, The weak scale from BBN, JHEP 12 (2014) 134 [arXiv: 1409.0551] [INSPIRE].

[51] R. Harnik, G.D. Kribs and G. Perez, A universe without weak interactions, Phys. Rev. D 74 (2006) 035006 [hep-ph/0604027] [INSPIRE].

[52] B.A. Campbell and K.A. Olive, Nucleosynthesis and the time dependence of fundamental couplings, Phys. Lett. B 345 (1995) 429 [hep-ph/9411272] [INSPIRE].

[53] E.J. Weinberg and A.-q. Wu, Understanding complex perturbative effective potentials, Phys. Rev. D 36 (1987) 2474 [INSPIRE].

[54] C. Ford, I. Jack and D.R.T. Jones, The standard model effective potential at two loops, Nucl. Phys. B 387 (1992) 373 [Erratum ibid. B 504 (1997) 551] [hep-ph/0111190] [INSPIRE].

[55] S.P. Martin, Three-loop standard model effective potential at leading order in strong and top Yukawa couplings, Phys. Rev. D 89 (2014) 013003 [arXiv:1310.7553] [InSPIRE].

[56] G. Degrassi et al., Higgs mass and vacuum stability in the Standard Model at NNLO, JHEP 08 (2012) 098 [arXiv: 1205.6497] [INSPIRE].

[57] Particle Data Group collaboration, M. Tanabashi et al., Review of particle physics, Phys. Rev. D 98 (2018) 030001 [INSPIRE].

[58] S.P. Martin and D.G. Robertson, Higgs boson mass in the standard model at two-loop order and beyond, Phys. Rev. D 90 (2014) 073010 [arXiv: 1407.4336] [INSPIRE].

[59] S.P. Martin and D.G. Robertson, TSIL: a program for the calculation of two-loop self-energy integrals, Comput. Phys. Commun. 174 (2006) 133 [hep-ph/0501132] [INSPIRE].

[60] H. Ooguri, E. Palti, G. Shiu and C. Vafa, Distance and de Sitter conjectures on the swampland, arXiv:1810.05506 [INSPIRE].

[61] E.J. Copeland, A.R. Liddle and D. Wands, Exponential potentials and cosmological scaling solutions, Phys. Rev. D 57 (1998) 4686 [gr-qc/9711068] [INSPIRE].

[62] P.G. Ferreira and M. Joyce, Cosmology with a primordial scaling field, Phys. Rev. D 58 (1998) 023503 [astro-ph/9711102] [INSPIRE]. 